\title{
HIGH-PERFORMANCE METHOD FOR CONSTRUCTING MEMBERSHIP FUNCTIONS BASED ON THE FUZZY CELLULAR SELF-ORGANIZING NEURAL NETWORKS
}

\section{S. V. GORBACHEV}

National Research Tomsk State University

Tomsk

Russia

e-mail: spp03@sibmail.com

\begin{abstract}
The article is devoted to the actual questions of the initialization of membership functions of fuzzy sets in adaptive systems analysis and fuzzy inference. Presents an analysis of methods for constructing such functions. Proposed highperformance neural network method based on new hybrid structures - fuzzy self-organizing Kohonen cellular neural networks (FCNN-SOM), allowing to automatize the process of fuzzification of the input variables and characterized by a high degree of self-organization of neurons, the lack of heuristic parameters of learning, the ability to selectively control individual connections between neurons to solve the problem of "dead" neurons and improved separating properties of the network in the case of overlapping clusters.
\end{abstract}

\section{Introduction}

In modern studies on the development of fuzzy methods of multidimensional evaluation of the effectiveness of innovative projects Keywords and phrases: technology, innovative projects, expert data, membership functions, fuzzy self-organizing cellular networks.

Received March 26, 2017

(C) 2017 Scientific Advances Publishers 
and technologies relevant issue is the initialization of membership functions of fuzzy sets $[1,2]$.

The construction of classical adaptive fuzzy inference systems ANFIS involves the formalization of the input parameters and target indicators of efficiency of innovative projects and technologies in the form of a vector of interval values (fuzzy interval), the hit in each interval which is characterized by some degree of uncertainty [3]. This procedure is called "fuzzification". Based on the source of information, experience and intuition of the experts often are confident enough to quantify the bounds (intervals) of possible (allowed) values of the parameters and their most probable (preferred) values. For example, the initial data can use the socalled triangular fuzzy number with membership function $\mu_{A}(x)$ of fuzzy set $A$ in the following form (Figure 1):

$$
\mu_{A}(x, a, b, c)=\left\{\begin{array}{lll}
0 & \text { if } & x \leq a, \\
\frac{x-a}{b-a} & \text { if } & a \leq x \leq b, \\
\frac{c-x}{c-b} & \text { if } & b \leq x \leq c, \\
0 & \text { if } & x \geq c .
\end{array}\right.
$$

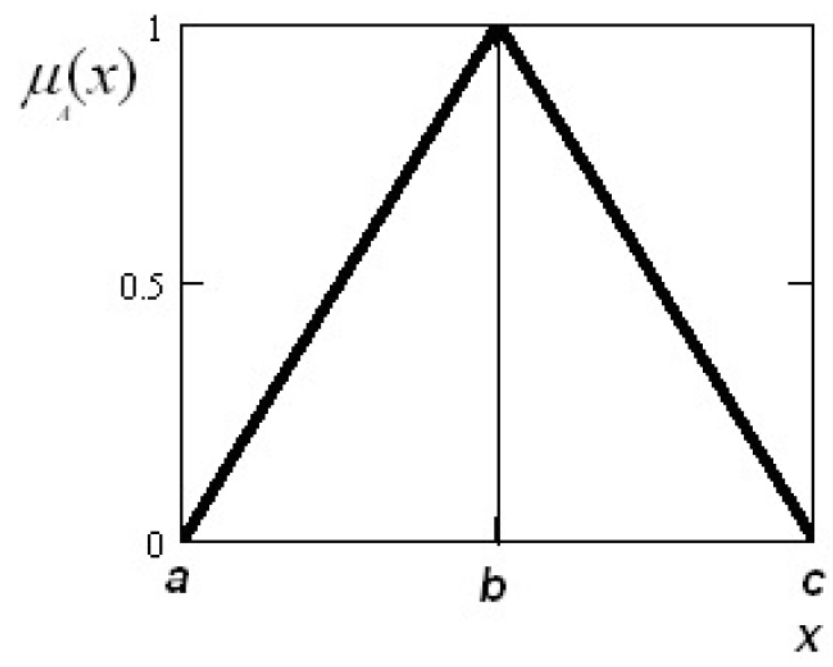

Figure 1. The membership function of triangular fuzzy number $A$. 
These numbers model the statement is the following: "parameter $A$ is approximately equal $b$ and unambiguously is in the range $[a ; c]$ ".

However, experts in some cases it is difficult to correctly define a set of membership functions. In this case, in practice, often used analytical representation of membership functions [4]. In Figure 2 shows the discrete approximation of some continuous Gaussian membership function:

$$
\mu(x)=\frac{1}{\sigma \sqrt{2 \pi}} e^{-\frac{(x-c)^{2}}{2 \sigma^{2}}}, \text { where } c \text { and } \sigma-\text { center and the standard }
$$

deviation (width).

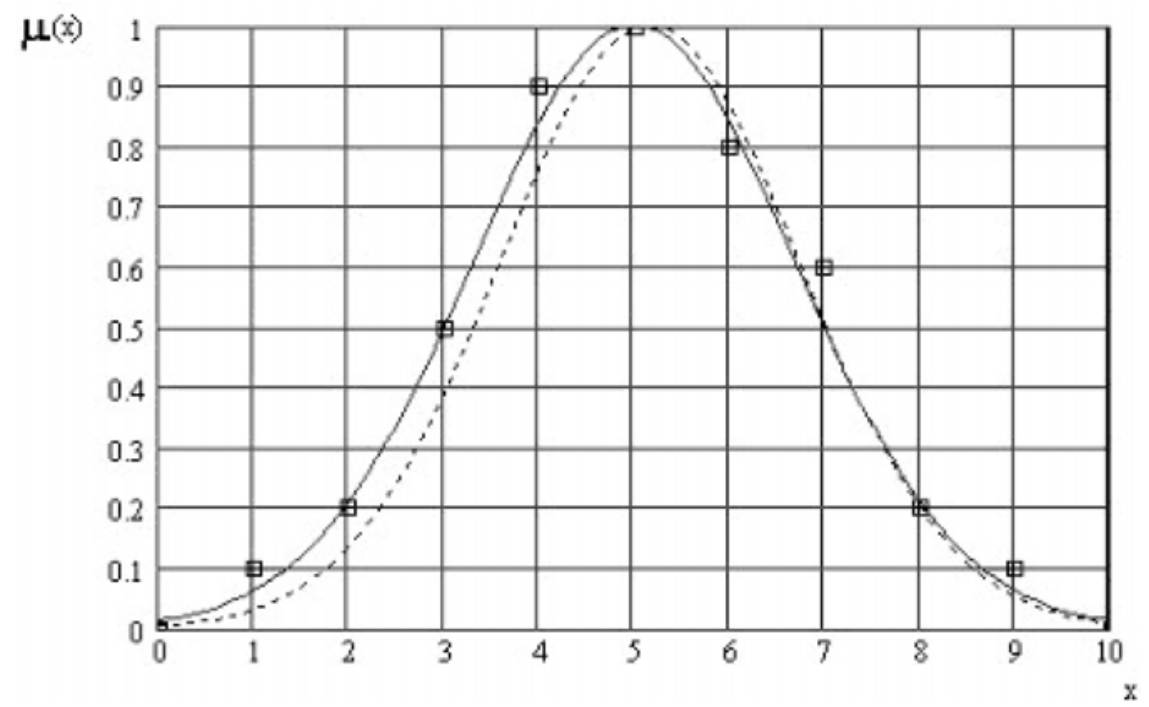

Figure 2. Gaussian (continuous) membership function, approximating discrete series.

Note that the definition of a fuzzy set does not impose restrictions on the choice of membership function [5], and in practice use many other membership functions that are defined as compositions of basic functions (double Gaussian, double sigmoidal, etc.), or as a combination of portions of ascending and descending (sigmoidal-Gaussian, spline, triangular, etc.). 


\section{Classification of Methods for Constructing of Membership Functions}

In the general case, methods for constructing membership functions can be divided into direct and consequential [6]. Direct methods include:

(1) direct the group;

(2) tabular method;

(3) the graphic method;

(4) the formula method.

The consequential methods include:

(1) method of statistical data;

(2) the method of paired comparisons;

(3) the method of expert evaluations;

(4) parametric method;

(5) the method of interval estimates;

(6) method of rank estimates.

Direct methods are characterized by the fact that the expert directly defines the rules for determining the values of the membership function. Direct methods of specifying the membership functions are used to measurable concepts such as speed, time, distance, pressure, temperature, etc., or when the allocated polar values. In this case, perhaps direct assignment of the membership function table, graph or formula. The varieties of direct methods include group methods, when, for example, the group of experts present specific object, and each examiner should give one of two answers: belongs or not this object to the specified set. Then the ratio of the number of affirmative answers to the total number of experts, and giving the value of the membership function of the object to the given fuzzy set [3]. From the analysis of the results of the research and solution of practical tasks presented in the literature, it is known that direct methods are mainly used as auxiliary, since they are characterized by a high degree of subjectivity. 
Consequential methods of creation of values of membership function are used in cases when there are basic measurable properties of the object, which are defined via fuzzy sets. In this case, the values of membership function are chosen so as to satisfy pre-defined conditions. Additional conditions may be imposed as a type of the received information and the procedure of its processing. These methods include statistical method, method of paired comparisons, method of expert estimations.

In the opinion of the paper author, the analytical task of the membership functions in solving semi-structured task imposes on the model of fuzzy inference to certain limitations and deficiencies that are the result formulated by the founder of the theory of fuzzy sets Zadeh "principle of incompatibility": “...the closer we come to solving real-world problems, it is clear that by increasing the complexity of the system our ability to make accurate and confident conclusions about its behaviour decreases until a certain threshold, for which the accuracy and confidence become almost mutually exclusive concepts" [7].

Meanwhile, the use of combinations of neural networks and fuzzy logic allows to obtain a synergistic effect [1] (Synergy - the total effect of the interaction of two or more factors, characterized by the fact that their effect is significantly greater than the effect of each individual component). The most important advantage of neural networks is the possibility of learning and adaptation. We don't have full knowledge about the object of analysis, such as mathematical model - based on the input and the desired (reference) signal, the neural network can learn to approximate functions of the object. Neural networks consist of a large number of interconnected simple processing elements (neurons), which results in the huge computational power when using parallel processing. On the other hand, the accumulated neural network knowledge are distributed among all its elements, making them inaccessible to the observer [8]. This deficiency deprived of fuzzy inference system that makes decisions based on rules recorded in the form of implication "If..., then". The unification of both approaches allows, on the one hand, to bring the learning ability and computational power of neural networks 
into fuzzy inference system, and on the other hand is to strengthen the intellectual capabilities of neural networks intrinsic to the human way of thinking fuzzy rules to reach a solution.

In this case, neuro-fuzzy networks are used to study system behaviour, based on input and output data and to generate fuzzy rules and membership functions. Comprehensive application of fuzzy logic and neural networks minimizes the system cost while increasing its efficiency. In Figure 3 shows an example of a 5-layer adaptive network of fuzzy inference (ANFIS) that implements the algorithm of fuzzy inference of Takagi-Sugeno with 2 input variables $\left(x_{1}\right.$ and $\left.x_{2}\right)$ and 2 fuzzy rules for the 2-th layer. For linguistic evaluation of the input variables $x_{1}$ and $x_{2}$ in the 2 -th layer (fuzzification) is used 2 terms.

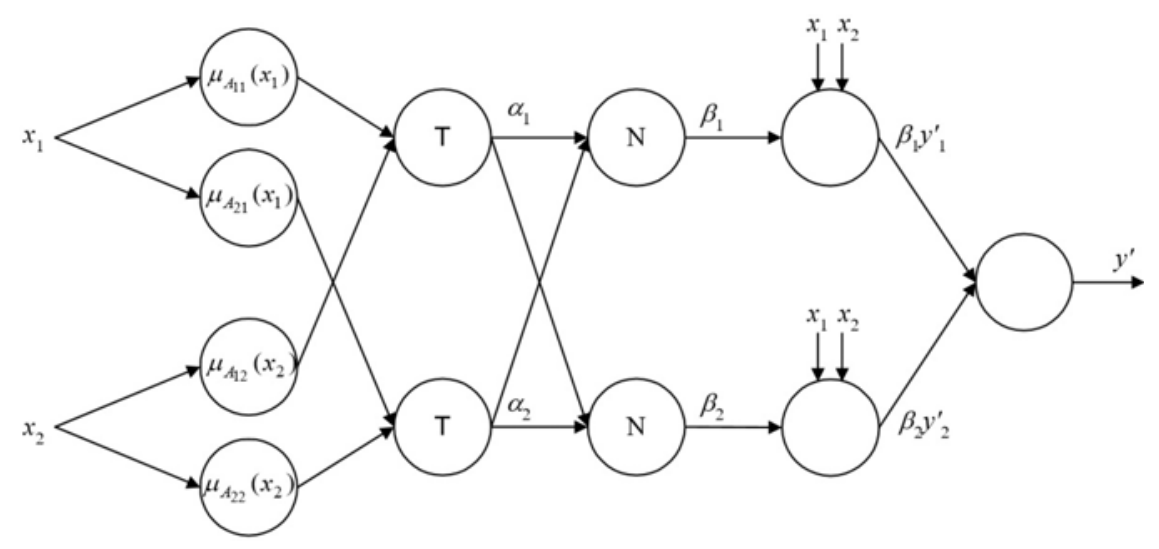

Figure 3. An example of the ANFIS network that implements the algorithm of fuzzy inference of Takagi-Sugeno.

The purpose of this study is to develop a high-performance neural network approach of fuzzification of the source data is poorly formalized and its experimental verification.

Note that the attributes of innovative projects or technologies can be classified as subjective or objective. Subjective quality characteristics include, for example, the technical level, innovation risk, project management - will evaluate their linguistic terms represented by fuzzy numbers based on expert assessments. 
Objective (quantitative) traits include, for example, the project investment costs etc. These quantitative features need to be brought to a common scale to ensure compatibility with the linguistic values of the subjective characteristics.

\section{Fuzzification of the Input Variables}

Fuzzification involves the translation of numerical attribute values into linguistic terms in order to reduce the information and presenting it in a human-understandable form for decision-making. The author's proposed approach involves the use of Kohonen self-organizing maps [9] to cluster the original data to find centers of membership functions [1].

Let the $i$-th attribute of the analyzed innovative projects (technologies) contains a data set $X$ with dimension $N$ (where $N$ is the number of analyzed objects) that need to be translated into $k$ linguistic variables $T_{j}, j=1,2, \ldots, k$. For simplicity, assume that the function $T_{j}$ has the form of triangulation:

$$
\begin{aligned}
& T_{1}(x)=\left\{\begin{array}{cl}
1, & x \leq a_{1} ; \\
\left(a_{2}-x\right) /\left(a_{2}-a_{1}\right), & a_{1}<x<a_{2} ; \\
0, & x \geq a_{2}, \\
0, & x<a_{j-1} ;
\end{array}\right. \\
& T_{j}(x)=\left\{\begin{array}{cl}
\left(x-a_{j-1}\right) /\left(a_{j}-a_{j-1}\right), & a_{j-1} \leq x \leq a_{j} ; \\
\left(a_{j+1}-x\right) /\left(a_{j+1}-a_{j}\right), & a_{j} \leq x \leq a_{j+1} ; \\
0, & x>a_{j+1}, \\
1, & x \geq a_{k} ;
\end{array}\right. \\
& T_{k}(x)=\left\{\begin{array}{cc}
\left(x-a_{k-1}\right) /\left(a_{k}-a_{k-1}\right), & a_{k-1}<x<a_{k} ; \\
0, & x \leq a_{k-1} .
\end{array}\right.
\end{aligned}
$$


Parameters to be determined for each attribute, form $k$ centers $\left\{a_{1}, a_{2}, \ldots, a_{k}\right\}$. The Kohonen network uses the following model (Figure 4): it consists of $M$ neurons that form the output of the Kohonen layer in the form of a rectangular grid on the plane.

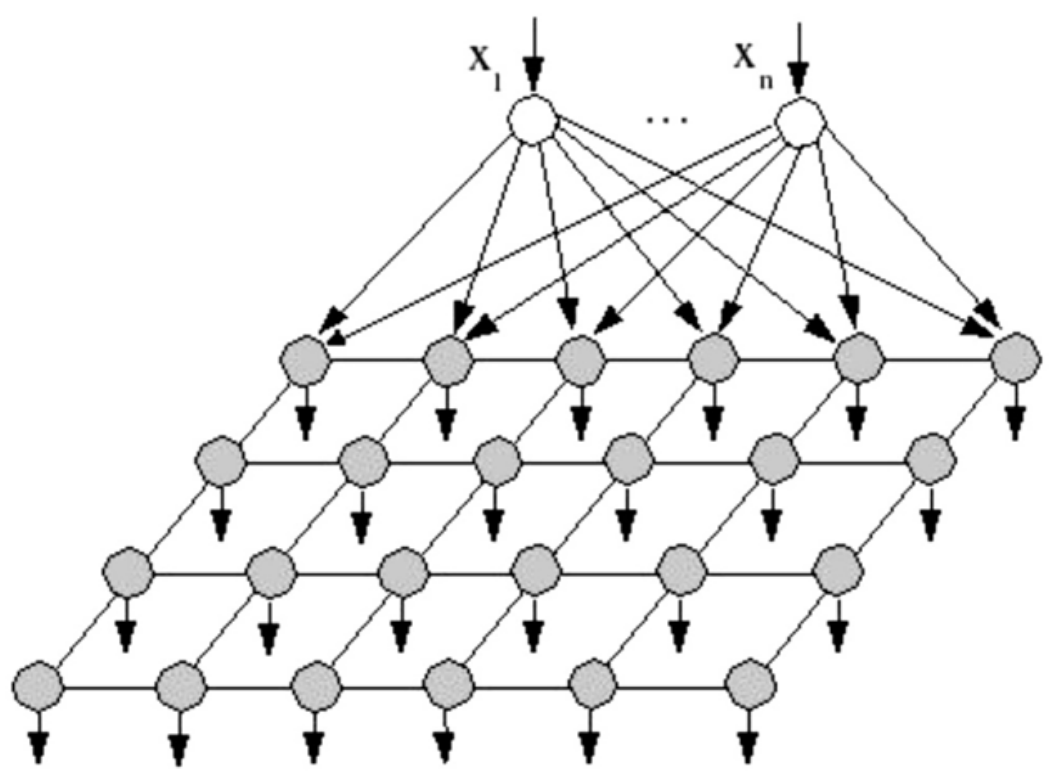

Figure 4. The model of Kohonen network.

The neurons located in the output layer, are suitable synapses, which receives $N$-dimensional input signal. Each neuron is characterized by its position in the layer and the weighting coefficient.

The Kohonen network is trained by successive approximations. In the process of learning the inputs served by data, but the network is not adjusted under the reference output value, and under patterns in the input data. Training begins with randomly chosen output location centers.

In the process of sequential feeding to the input of the network training examples determines the most similar neuron (the one with the dot product of the weights and an input vector is minimal). This neuron 
is declared the winner and is the center of adjusting weights of the neighboring neurons. This learning rule implies a "competitive" training with the distance of the neurons from the "winning neuron".

This education is not to minimize error and adjust weights (internal parameters of the neural network) for most matches with the input data.

After the presentation of a sufficient number of input vectors of synaptic weights of the network acquire the ability to identify clusters. Weights are organized so that topologically close nodes become sensitive to similar inputs.

The result of the algorithm, the cluster center is set at a specific position to satisfactorily cluster examples for which this neuron is the "winner".

The disadvantages of such a "competitive" training of SOM are:

(1) the use of heuristic parameters and processes to solve the problem of "dead" neurons, leading to increased training time;

(2) the impossibility of separating of overlapping clusters.

\section{The Development of Hybrid Fuzzy Cellular Self-organizing Neural Networks}

The first studies of cellular neural networks (CNN) was conducted by Chua and Yang [10, 11], mainly in computational models of image processing. In [12] proved the possibility of learning of Kohonen's selforganizing neural networks by cellular automat that can significantly improve the quality and speed of learning, however, not resolved the issues dividing the network properties in the case of overlapping clusters.

The author proposed architecture of fuzzy cellular self-organizing networks (FCNN-SOM) contains 3 layers: neural:

(1) input (receptor) layer;

(2) the layer of Kohonen neurons with lateral connections, trainees by cellular automat, to identify centroids of overlapping clusters; 
(3) additional (output) layer of fuzzy clustering (Figure 5) calculating levels of current vector for each cluster.

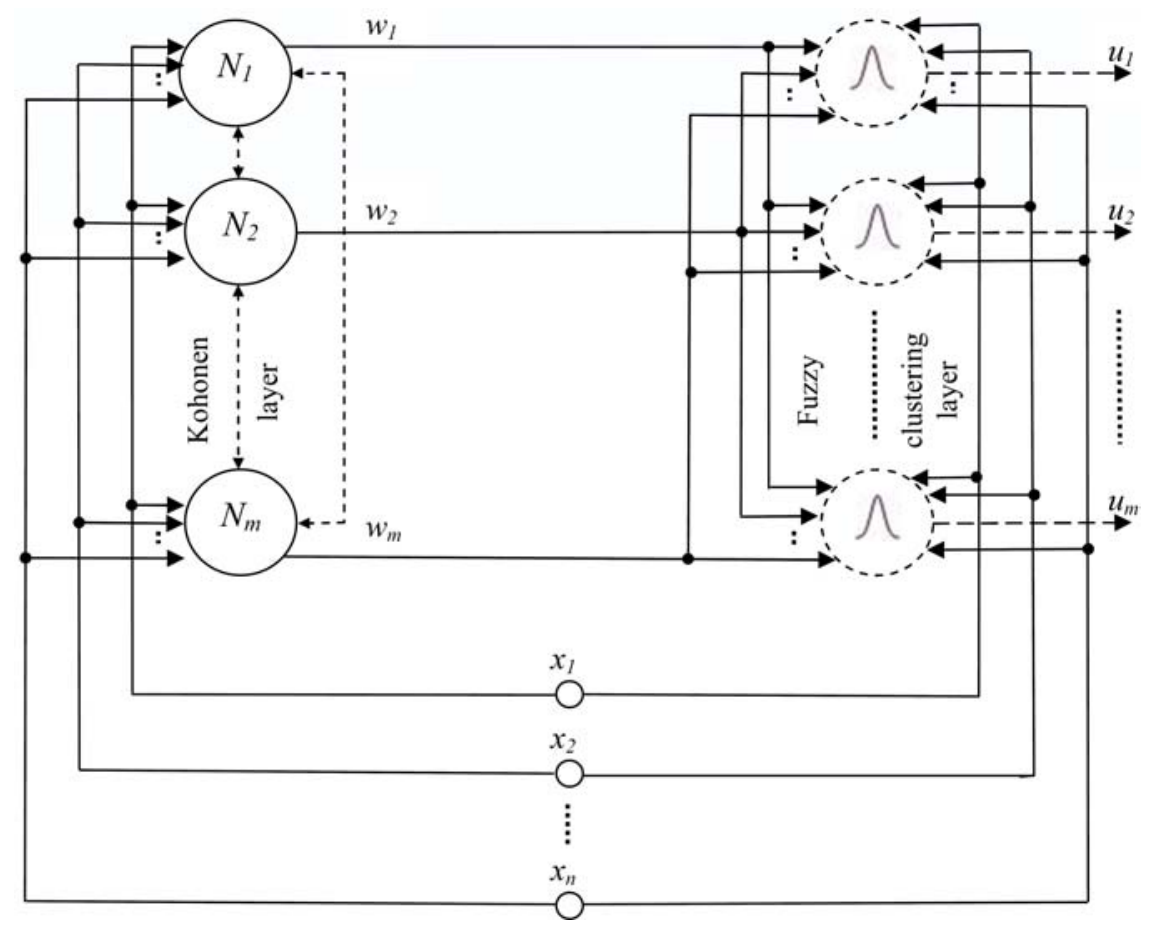

Figure 5. The architecture of fuzzy cellular self-organizing neural networks (FCNN-SOM).

The training of FCNN-SOM consists of the following steps.

\section{The Learning Algorithm of Fuzzy Cellular Self-organizing Neural Networks}

Source data - sample of observations, formed from $n$-dimensional feature vectors $x(k), k=1, \ldots, N$.

The purpose of education - division of learning sample data into $m$ clusters with some levels $w_{j}(k)$ of membership of $k$-th feature vector to the $j$-th cluster $(j=1, \ldots, m)$. 
Step 1. Data pre-processing - the input data is centered and standartisied on all components of the vector so that all observations belonged to hypercube $[-1 ; 1]^{n}$.

Step 2. Initialization of the primary state of neural network, which provides self-adaptive regulation of neuronal network with the formation of an ordered self organizing Kohonen maps. The main idea of this method is described in [12] and consists in the gradual retraction by the active neurons of inactive neurons to the normalized hypercube space of the training samples. For this, initialization of the network, all the neurons of the source placed at an arbitrary point on hypersphere of sufficiently large radius $R$, whose center coincides with the center of space of the training samples. The radius $R$ of the hypersphere is selected so that under hypersphere neurons could never win and "involved" in an hypercube of sample only through its relationship with the previously winning neuron, but not at the expense of their own victories.

Step 3. Training of the Kohonen layer by Moore's cellular automat on A simply-connected or multiply-connected SOM [12].

The cell of the cellular automaton is the neuron of the Kohonen layer, topologically connected in the environment of Moore with 8 (on the border - with 3 or 5) neighbuoring neurons of the two-dimensional selforganizing map of Kohonen (Figure 6). 

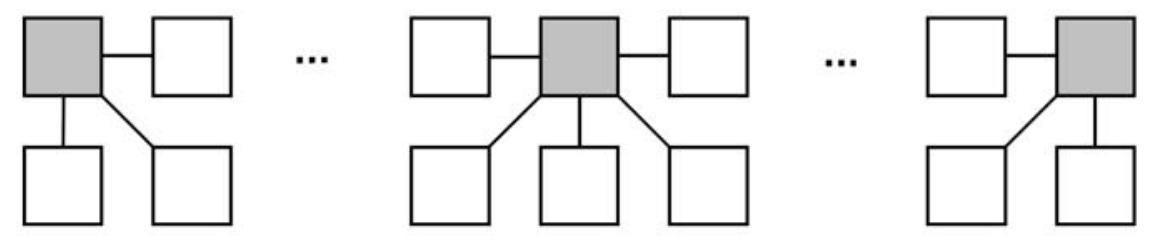

$\cdots$
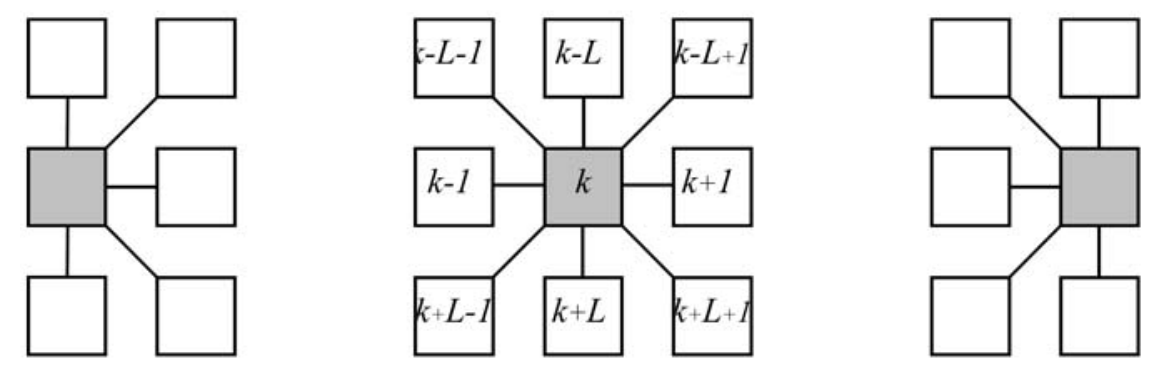

...
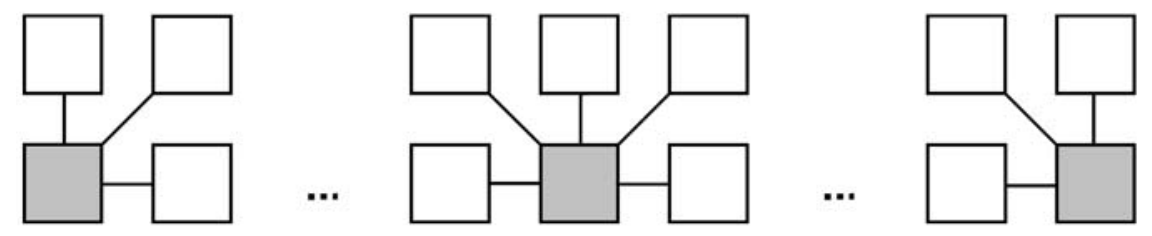

Figure 6. The topology of the Kohonen cell map, surrounded by Moore.

\section{Experimental Evaluation of the Accuracy of the Developed Method}

We calculated the accuracy of the developed method by crosschecking. To this end, according to the learning sample, various ANFIS networks with the corresponding membership functions were built, and their ability to predict was evaluated on a test sample. Cross-checking is the procedure for evaluating the accuracy of classification on data from a test set, which is also called a crossvalidation set. The accuracy of the classification of the test set is compared with the accuracy of the classification of the training set. If the classification of the test set yields 
approximately the same accuracy results as the classification of the training set, it is considered that the model passed a cross-check. The division into training and test sets was carried out by dividing the sample in a certain proportion, for example, the training set - $80 \%$ and the test set - $20 \%$. The evaluation of the probability of the correct classification of test samples by the ANFIS network, depending on the type of membership function, was performed on the quality functional:

$$
E=\frac{N_{R}}{N_{T}}
$$

where $N_{T}$ is the number of samples of the test sample, $N_{R}$ is the number of matches of classification results with real data.

Based on the results of the evaluation of the reliability of the results of fuzzy inference, Table 1 was compiled, which shows the values $E_{i}$ of the classification for each efficiency class of the test samples. The best result is achieved for an adaptive network with initialization of membership functions using the developed FCNNSOM method, for which the average estimate of the reliability of the classification is $E=0.9$. 
Table 1. Results of assessing the reliability of classification results adaptive network of fuzzy inference

\begin{tabular}{|c|c|c|c|}
\hline $\begin{array}{l}\text { The method of } \\
\text { initializing } \\
\text { membership } \\
\text { functions }\end{array}$ & Efficiency class & $E_{i}$ & $E$ \\
\hline $\begin{array}{c}\text { Classical with } \\
\text { Gaussian functions }\end{array}$ & $\begin{array}{c}\text { high } \\
\text { average } \\
\text { low }\end{array}$ & $\begin{array}{l}0.83 \\
0.61 \\
0.59\end{array}$ & 0.68 \\
\hline $\begin{array}{c}\text { Classic with triangular } \\
\text { functions }\end{array}$ & $\begin{array}{c}\text { high } \\
\text { average } \\
\text { low }\end{array}$ & $\begin{array}{l}0.81 \\
0.67 \\
0.53\end{array}$ & 0.67 \\
\hline Linear interpolation & $\begin{array}{c}\text { high } \\
\text { average } \\
\text { low }\end{array}$ & $\begin{array}{l}0.77 \\
0.58 \\
0.42\end{array}$ & 0.59 \\
\hline $\begin{array}{c}\text { Piece Approximation } \\
\text { Gaussian features }\end{array}$ & $\begin{array}{c}\text { high } \\
\text { average } \\
\text { low }\end{array}$ & $\begin{array}{l}0.85 \\
0.68 \\
0.64\end{array}$ & 0.72 \\
\hline $\mathrm{SOM}$ & $\begin{array}{c}\text { high } \\
\text { average } \\
\text { low }\end{array}$ & $\begin{array}{l}0.92 \\
0.87 \\
0.81\end{array}$ & 0.87 \\
\hline FCNN-SOM & $\begin{array}{c}\text { high } \\
\text { average } \\
\text { low }\end{array}$ & $\begin{array}{l}0.95 \\
0.89 \\
0.86\end{array}$ & 0.9 \\
\hline
\end{tabular}

\section{Conclusion}

The developed method of constructing membership functions based on a new hybrid architecture - fuzzy cellular self-organizing maps of Kohonen (FCNN-SOM) - allows to automate the procedure of fuzzification in adaptive systems of fuzzy inference and place the centers of membership functions in such a way that they cover only those areas of input and output spaces. The use of FCNN-SOM provides: 
- high speed of calculations due to parallelism;

- a high degree of self-organization of neurons;

- lack of heuristic learning parameters;

- the possibility of selective management of individual connections between neurons to solve the problem of "dead" neurons;

- improvement of the separating properties of the network in the case of overlapping clusters;

- significant resistance to fluctuations in parameters;

- the necessary accuracy and adaptability, training and self-organization.

In conclusion, we note that such hybrid neural-fuzzy networks have great potential from the point of view of creating intellectual systems for analyzing, managing and supporting decision-making in the case of processing multidimensional weakly formalized data describing innovative objects and the trajectory of scientific, technological and innovative development.

\section{Acknowledgements}

The research was performed under the program of competitiveness increase of National Research Tomsk State University among the world's leading scientific and educational centers, with the financial support of Russian Foundation for Basic Research, grant № 16-29-12858.

\section{References}

[1] S. V. Gorbachev and V. I. Syryamkin, Neuro-fuzzy methods in intellectual systems for processing and analyzing multidimensional information, Tomsk: Tomsk State University (2014), 510.

[2] Janikow, Fuzzy Processing in Decision Trees, Proceedings of the Sixth International Symposium on AI (1993), 360-367.

[3] V. V. Boyarshinova and D. K. Eltyshev, Integrated intellectual assessment of the state of electrical equipment, Automated control systems and information technologies: Materials Rus. Scientific-practical. Conf., Perm, May 15, 2015 - Perm: Publishing House of the Central Scientific and Technical University (2015), 243-248. 
[4] S. V. Gorbachev and V. I. Syryamkin, Adaptive neuro-fuzzy recognition technology intersecting objects, Applied Mechanics and Materials, Volume 756 (Mechanical Engineering, Automation and Control Systems) (2015), 683-688.

[5] V. G. Rubanov, Intellectual systems of automatic control, Unclear management in technical systems: Textbook, V. G. Rubanov and A. G. Filatov; Publishing House BSTU (2010), 170.

[6] A. Kaid, Methods for constructing the fuzzy affiliation of fuzzy sets, Izvestiya Southern Federal University, Technical Sciences №. 2(139) (2013), 144-152.

[7] L. A. Zadeh, Fuzzy sets, Information and Control 8(3) (1965), 338-353.

[8] D. Rutkovskaya, M. Pilinsky and L. Rutkowski, Neural networks, genetic algorithms and fuzzy systems, Moscow (2006), 383.

[9] T. Kohonen, Self-Organizing Maps (Third Extended Edition), New York (2001), 501.

[10] L. O. Chua and L. Yang, Cellular neural networks: Theory, IEEE Transactions on Circuits and Systems CAS-35(10) (1988), 1257-1272.

[11] L. O. Chua and L. Yang, Cellular neural networks: Applications, IEEE Transactions on Circuits and Systems CAS-35(10) (1988), 1273-1290.

[12] V. I. Anikin, Training of artificial neural network Kohonen cellular automaton, V. I. Anikin and A. A. Karmanov; Information Technology 11 (2014), 73-80. 